\title{
Educação alimentar na obesidade: adesão e resultados antropométricos
}

\author{
Food education for the obese: adherence \\ and anthropometric results
}

Júlia Macedo BUENO ${ }^{1}$

Francine Scochi LEAL ${ }^{1}$

Luciana Pereira Lima SAQUY'

Claudia Benedita dos SANTOS 2

Rosane Pilot Pessa RIBEIRO²

RE S U M O

\section{Objetivo}

O objetivo deste estudo foi avaliar a adesão de indivíduos com excesso de peso a um programa de educação alimentar e a evolução antropométrica, no período de março de 2005 a dezembro de 2007.

\section{Métodos}

A amostra foi composta por adultos de uma comunidade universitária pública, de ambos os sexos, na faixa etária entre 20 e 59 anos. Durante 10 semanas, em grupo, eles receberam orientação alimentar e apoio psicológico, e praticaram exercício físico adaptado. Foram coletados dados sociodemográficos, de peso e de estatura, para cálculo do índice de massa corporal, além da circunferência da cintura no início e no final da intervenção.

\section{Resultados}

Como resultados, 116 indivíduos com idade de 41 anos inscreveram-se no programa, a maioria do sexo feminino $(73,3 \%)$, casada $(70,7 \%)$ e com ensino médio completo $(49,1 \%)$. Houve adesão de $50,0 \%$ deles. Os desistentes eram obesos (65,5\%), com índice de massa corporal médio de $33,4 \mathrm{~kg} / \mathrm{m}^{2}$. Aqueles que completaram o programa apresentaram queda no índice de massa corporal $(32,5$ para 31,2kg/m²) com perda ponderal média de 3,9\%; $37,9 \%$ apresentaram perda maior ou igual a 5,0\%. A circunferência da cintura também reduziu de 102,1 para $98,6 \mathrm{~cm}$.

\section{Conclusão}

Conclui-se que a adesão foi baixa, mas a evolução antropométrica daqueles que aderiram à estratégia foi satisfatória, sugerindo que a combinação de ações multi- e interdisciplinares podem ser eficientes para o tratamento da obesidade.

Termos de indexação: Cooperação do paciente. Educação alimentar e nutricional. Obesidade. Perda de peso.

1 Universidade de São Paulo, Escola de Enfermagem de Ribeirão Preto, Programa de Reeducação Alimentar. Ribeirão Preto, SP, Brasil.

2 Universidade de São Paulo, Escola de Enfermagem de Ribeirão Preto, Departamento de Enfermagem Materno-Infantil e Saúde Pública. Av. Bandeirantes, 3900, 14040-900, Ribeirão Preto, SP, Brasil. Correspondência para/Correspondence to: R.P.P. RIBEIRO E-mail: <rribeiro@eerp.usp.br>. 
576 | J.M. BUENO et al.

\section{A B S T R A C T}

\section{Objective}

The goal of this study was to evaluate the adherence of overweight subjects to a food education program and their anthropometric history from March, 2005 to December, 2007.

\section{Methods}

Males and females aged 20 to 59 years from a public university community were included. The group received dietary advice, psychological support and performed special physical activities over a period of 10 weeks. Sociodemographic information, weight, height and waist circumference were collected to calculate body mass index at baseline and end of the program.

\section{Results}

One-hundred and sixteen 41-year old subjects enrolled in the program; most of them were married (70.7\%), had a high school diploma (49.1\%), and were females (73.3\%). Half the subjects (50.0\%) adhered to the program. The defaulters (65.5\%) were overweight, with a mean body mass index of $33.4 \mathrm{~kg} / \mathrm{m}^{2}$. The body mass index of the participants who completed the program decreased from 32.5 to $31.2 \mathrm{~kg} / \mathrm{m}^{2}$, with an average weight loss of $3.9 \%$ of their body weight; $37.9 \%$ lost $\geq 5.0 \%$ of their body weight. Waist circumference also decreased from 102.1 to $98.6 \mathrm{~cm}$.

\section{Conclusion}

Although adherence was low, the anthropometric history of those who remained in the program was satisfactory, suggesting that a combination of multi- and interdisciplinary actions can be effective for treating obesity.

Indexing terms: Patient compliance. Food and nutrition education. Obesity. Weight loss.

\section{N T R O D U Ç Ã O}

A obesidade é reconhecidamente um problema de saúde que afeta as sociedades em diversas partes do mundo, caracterizando-se na atualidade como uma epidemia com tendência a pandemia, não apenas em países industrializados, como também naqueles em desenvolvimento'. A Organização Mundial da Saúde (OMS) estima que exista hoje um bilhão de pessoas com sobrepeso no mundo e mais de 300 milhões de indivíduos com obesidade 2 . No Brasil, segundo os dados da Pesquisa de Orçamento Familiar (POF), o excesso de peso afeta $41,1 \%$ dos homens e $40,0 \%$ das mulheres: a obesidade atingiu $8,9 \%$ dos homens e $13,1 \%$ das mulheres adultas ${ }^{3}$.

A etiologia da obesidade é complexa, multifatorial, resultante da interação genética e ambiental, mediada por fatores sociais, econômicos, endócrinos, metabólicos e psiquiátricos ${ }^{3}$. Consequentemente, o tratamento da obesidade é complexo e deve ser multiprofissional e interdisciplinar, pois, mais do que a simples redução de peso, ele deve visar a mudanças no estilo de vida, que devem ser mantidas para sempre ${ }^{4}$.
Sendo assim, há de se reservar lugar de destaque para ações de educação em nutrição e alimentação, com redução moderada na ingestão de energia como estratégia para redução da massa corporal. A associação da prática de exercícios físicos com as mudanças nos hábitos cotidianos pode alcançar, de forma eficaz, todas as camadas sociais da população. Essas estratégias também correspondem às principais formas de tratamento não farmacológico da obesidade².

A mudança nos hábitos de vida e, consequentemente, das questões alimentares não deve advir de um processo de normatização e muito menos de culpabilização do indivíduo, pois as modificações não se restringem apenas à mudança no consumo de alimentos e à atividade física, mas têm influência sobre toda a constelação de significados ligados ao comer, ao corpo e ao viver $^{5}$.

A educação alimentar tem um papel importante em relação ao processo de transformação, recuperação e promoção de hábitos alimentares saudáveis, pois pode proporcionar conhecimentos necessários à autotomada de 
decisão, formando atitudes, hábitos e práticas alimentares sadias e variadas 6 .

Como proposta dietética, a educação alimentar defende que é possível emagrecer e manter o peso comendo diversos alimentos, já que nada deve ser proibido, desde que respeitadas as quantidades estabelecidas previamente. Além de ser um método saudável, sensato e seguro, é voltado para a formação de valores, para o prazer, a responsabilidade, a atitude crítica, assim como para o lúdico e a liberdade. É um programa que deve ser seguido para sempre, já que prevê a aquisição e a incorporação de novos hábitos e comportamentos diante da alimentação. Devido a isso, o emagrecimento é mais lento quando comparado a outras dietas altamente restritivas, porém o resultado é mais duradouro ${ }^{7-9}$.

No entanto, para que a educação alimentar tenha os resultados esperados, é necessária a adesão do sujeito à terapêutica. Sendo a obesidade uma doença crônica, exige tratamentos de longa duração, que, em geral, apresentam menor adesão, visto que os esquemas terapêuticos exigem um grande empenho do paciente, que necessita modificar seus hábitos de vida para alcançar uma melhora do quadro9.

A Organização Mundial da Saúde (OMS) adota como definição de adesão a tratamentos crônicos uma fusão de outras duas definições, de Haynes $^{10}$ e Rand ${ }^{11}$, que conceituam adesão como o grau em que o comportamento de uma pessoa - representado pela ingestão de medicação ou pelo seguimento da dieta ou pelas mudanças no estilo de vida - corresponde e concorda com as recomendações de um médico ou outro profissional de saúde.

Diante dessas considerações, o objetivo deste estudo foi avaliar a adesão e a evolução antropométrica de indivíduos com excesso de peso e participantes de um programa de educação alimentar.

\section{MÉ T O D O S}

O estudo, com desenho retrospectivo, longitudinal, descritivo e quantitativo foi realizado com 116 indivíduos com excesso de peso que participaram do Programa de Reeducação Alimentar desenvolvido pela Escola de Enfermagem de Ribeirão Preto da Universidade de São Paulo (PRAUSP), no período de março de 2005 a dezembro de 2007. Os sujeitos eram de ambos os sexos, na faixa etária entre 20 e 59 anos. Os critérios de exclusão foram: gravidez, abandono do programa antes de seu término, mais do que $25 \%$ de faltas durante seu desenvolvimento e deficiência física que levasse a alterações no método antropométrico.

Esse projeto foi criado em 1998 e passou a ser desenvolvido a partir de 2005, no Centro Multidisciplinar de Promoção à Saúde e Prevenção de Doenças da Coordenadoria do Campus de Ribeirão Preto - USP (CCRP-USP), contando com uma equipe multidisciplinar composta por nutricionistas, psicóloga, educador físico e aluno do curso de graduação em enfermagem. A população atendida inicialmente originou-se das diversas seções e serviços da CCRP-USP e depois o programa foi aberto a toda comunidade do campus. Assim, nove grupos de pessoas com excesso de peso participaram do programa, desenvolvido ao longo de 10 semanas com um encontro semanal para orientação alimentar e apoio psicológico e três encontros semanais para realização de atividade física no Centro de Educação Física, Esportes e Recreação (CEFER) da USP - Ribeirão Preto.

A orientação alimentar baseou-se na discussão de um tema a cada encontro: pirâmide alimentar, grupos alimentares e porções, sugestão de cardápio, trocas alimentares saudáveis, mudanças comportamentais como mastigação, fracionamento da alimentação e ingestão de água, uso de alimentos dietéticos e light, fibras, gorduras e colesterol, leitura de rótulos, entre outros assuntos solicitados pelos participantes. A utilização do registro alimentar foi uma estratégia adotada para acompanhar as mudanças e as dificuldades encontradas, visando à discussão e às formas de enfrentamento.

Os interessados em participar do PRAUSP buscaram o programa de maneira voluntária. 
Após as inscrições dos interessados, os grupos foram formados de acordo com o grau de excesso de peso e as necessidades e anseios em comum dos participantes.

Todas as pessoas tiveram seus dados coletados mediante autorização por escrito fornecida no primeiro encontro do grupo pelo Termo de Consentimento Livre e Esclarecido. O projeto foi submetido e aprovado pelo Comitê de Ética em Pesquisa da Escola de Enfermagem de Ribeirão Preto - USP, protocolo nº 0939/2008.

Para caracterização da população, foram coletados dados referentes à idade, sexo, estado civil e nível de escolaridade. Para avaliação antropométrica, realizou-se a mensuração do peso no início e no término do programa, utilizando-se balança portátil digital eletrônica Welmy, de capacidade máxima de $200 \mathrm{~kg}$, mínima de $2 \mathrm{~kg}$ e precisão de $50 \mathrm{~g}$. A estatura no momento inicial foi aferida utilizando-se o antropômetro acoplado à balança, de altura máxima de $2 \mathrm{~m}$ e precisão de $0,1 \mathrm{~cm}$. Já a circunferência da cintura ao início e ao final do programa foi aferida com uma fita métrica inextensível. Todas as medidas foram realizadas de acordo com o Manual do Sistema de Vigilância Alimentar e Nutricional do Ministério da Saúde ${ }^{12}$. Essas medidas foram utilizadas para o cálculo do Índice de Massa Corporal (IMC) (peso e altura) e como preditoras de risco de complicações metabólicas associadas à obesidade, que foram categorizadas segundo os referenciais propostos pela World Health Organization².

O percentual de perda de peso foi avaliado pela subtração do peso inicial menos final, multiplicado por 100 e dividido pelo peso inicial. Esse resultado foi categorizado em: a) perda maior ou igual a $5 \%$ do peso inicial; b) redução menor que $5 \%$ do peso inicial; c) ausência de redução, correspondente à manutenção (peso atual, Desvio-Padrão - DP=1\%) ou ganho de peso (considerado como acréscimo maior que $1 \%$ no peso inicial).

Os dados obtidos foram organizados em um banco de dados utilizando-se a técnica da dupla verificação. No programa estatístico Statistical Package for the Social Sciences (SPSS), versão $16.1^{13}$, os dados foram processados e analisados de forma descritiva (valores médios - $\mathrm{M}$ e DP) e inferencial.

Para avaliar uma possível diferença entre as médias inicial e final de IMC e circunferência da cintura, foi utilizado o teste $t$ de Student pareado. Utilizou-se a correlação de Pearson para correlacionar estado nutricional inicial com percentual de perda de peso e melhora no IMC e na circunferência da cintura. $O$ nível de significância utilizado foi $\alpha=0,05$.

\section{RES ULTA D OS}

Foram analisados como média e desvio-padrão os dados iniciais de 116 indivíduos adultos, com idade de $\mathrm{M}=41, \mathrm{DP}=9$ anos, que participaram do PRAUSP; 73,3\% eram do sexo feminino $(n=85)$ e $26,7 \%$ do sexo masculino $(\mathrm{n}=31)$ (Tabela 1).

Durante o transcorrer dos grupos, houve adesão de $50 \%$ dos sujeitos ao programa, entendida neste estudo como a permanência do indivíduo até o último encontro, com faltas que não ultrapassassem 25\%.

Os indivíduos que não preencheram esse critério foram considerados desistentes: a idade

Tabela 1. Distribuição dos participantes do PRAUSP, segundo variáveis sociodemográficas. Ribeirão Preto (SP), 2009.

\begin{tabular}{lcc}
\hline \multirow{2}{*}{ Variáveis sociodemográficas } & \multicolumn{2}{c}{ Participantes } \\
\cline { 2 - 3 } & $\mathrm{n}$ & $\%$ \\
\hline Estado civil & & \\
Casado ou união estável & 82 & 70,7 \\
Solteiro & 22 & 19,0 \\
Viúvo & 2 & 1,7 \\
Divorciado & 10 & 8,6 \\
Nível de escolaridade & & \\
Ensino Fundamental Incompleto & 4 & 3,4 \\
Ensino Fundamental Completo & 9 & 7,8 \\
Ensino Médio Incompleto & 5 & 4,3 \\
Ensino Médio Completo & 57 & 49,1 \\
Ensino Superior Incompleto & 16 & 13,8 \\
Ensino Superior Completo & 25 & 21,6 \\
\hline
\end{tabular}

PRAUSP: programa de reeducação alimentar desenvolvido pela Escola de Enfermagem de Ribeirão Preto da Universidade de São Paulo. 
era de $\mathrm{M}=39, \mathrm{DP}=10$ anos, a maioria do sexo feminino $(70,7 \%, n=41)$, casada $(72,4 \%, n=42)$ e aproximadamente a metade tinha o ensino médio completo $(48,3 \%, \mathrm{n}=28$ ) (Tabela 2 ).

Em relação ao estado nutricional, os sujeitos que desistiram do programa encontravam-se em sua maioria obesos, com IMC de $33,4 \mathrm{Kg} / \mathrm{m}^{2}$, classificado como obesidade grau I. Por categoria desse parâmetro, 34,5\% deles tinha sobrepeso, $31 \%$ obesidade grau I, $22,4 \%$ obesidade grau II e $12,1 \%$ obesidade grau III.

Os participantes que permaneceram até o final do programa eram, na maioria, do sexo feminino ( $n=44,75,9 \%)$, casados $(n=40,69 \%)$ e metade deles tinha o ensino médio completo $(n=29)$.

A partir das medidas antropométricas coletadas ao início e ao final do programa, observou-se que as médias de IMC foram $M=32,5$, $\mathrm{DP}=5,7 \mathrm{Kg} / \mathrm{m}^{2}$ e $\mathrm{M}=31,2, \mathrm{DP}=5,6 \mathrm{Kg} / \mathrm{m}^{2}$, respectivamente, caracterizando condição de obesidade em ambos os casos. Mesmo mantendo-se a mesma classe de IMC, houve diferença estatística entre essas médias $(p=0,00)$, o que indica melhora nos padrões antropométricos. Percebeu-se, ainda, que no início do programa, 39,7\% dos sujeitos apresentavam sobrepeso $(n=23)$ e $60,3 \%$ eram obesos ( $n=35)$. Já ao final, o percentual de não obesos (eutrofia $n=4$; sobrepeso $n=28$ ) foi de
$55,2 \%(n=32)$ e o de obesos foi reduzido para $44,8 \%(n=26)$. Na Figura 1, apresenta-se a divisão dos participantes do programa em relação ao estado nutricional inicial e final.

Quando comparadas as características sociodemográficas dos indivíduos que desistiram do programa com as daqueles que aderiram à estratégia, pôde-se notar que esses dois grupos não diferiram em relação às variáveis investigadas. No entanto, em relação à antropometria, apresentou-se porcentagem maior de indivíduos com obesidade graus II e III no grupo de desistentes quando comparada à porcentagem do grupo que permaneceu até o final dos encontros (Figura 2).

Em relação à redução de peso durante o programa, houve perda média de $\mathrm{M}=3,9, \mathrm{DP}=3 \%$ do peso inicial: $37,9 \%$ apresentaram perda maior ou igual a $5 \%(n=22), 44,8 \%$ perderam menos de $5 \%(n=26)$ e apenas $17,2 \%$ não apresentaram redução de peso $(n=10)$.

O estado nutricional inicial não influenciou o percentual de perda de peso dos indivíduos, pois não houve correlação $(r=0,03)$ estatisticamente significativa $(p=0,79)$ entre essas duas variáveis.

Pela análise da circunferência da cintura, as médias inicial e final da população foram $M=102,1 \mathrm{~cm}, D P=11,6 \mathrm{~cm}$ e $M=98,6, D P=11,8 \mathrm{~cm}$.

Tabela 2. Distribuição dos participantes do PRAUSP, desistentes ou não segundo variáveis sociodemográficas. Ribeirão Preto (SP), 2009.

\begin{tabular}{|c|c|c|c|c|}
\hline \multirow{3}{*}{ Variáveis sociodemográficas } & \multicolumn{4}{|c|}{ Participantes } \\
\hline & \multicolumn{2}{|c|}{ Desistentes } & \multicolumn{2}{|c|}{ Não desistentes } \\
\hline & $\mathrm{n}$ & $\%$ & $\mathrm{n}$ & $\%$ \\
\hline \multicolumn{5}{|l|}{ Estado civil } \\
\hline Casado ou união estável & 42 & 72,4 & 40 & 69,0 \\
\hline Solteiro & 11 & 19,0 & 11 & 19,0 \\
\hline Viúvo & 0 & 0 & 2 & 3,4 \\
\hline Divorciado & 5 & 8,6 & 5 & 8,6 \\
\hline \multicolumn{5}{|l|}{ Nível de escolaridade } \\
\hline Ensino Fundamental Incompleto & 1 & 1,7 & 3 & 5,2 \\
\hline Ensino Fundamental Completo & 5 & 8,6 & 4 & 6,9 \\
\hline Ensino Médio Incompleto & 3 & 5,2 & 2 & 3,4 \\
\hline Ensino Médio Completo & 28 & 48,3 & 29 & 50,0 \\
\hline Ensino Superior Incompleto & 11 & 19,0 & 5 & 8,6 \\
\hline Ensino Superior Completo & 10 & 17,2 & 15 & 25,9 \\
\hline
\end{tabular}


A primeira caracteriza risco muito elevado tanto para homens quanto para mulheres e a segunda, risco elevado para o sexo masculino e muito elevado para o sexo feminino. Houve redução estatisticamente significante entre essas médias $(p=0,00)$, ou seja, melhora desse parâmetro antropométrico.

A Figura 3 ilustra o percentual de indivíduos com alteração no risco relativo a complicações metabólicas associadas à medida da circunferência da cintura. Encontrou-se alta porcentagem de indivíduos que apresentavam risco muito elevado no início da estratégia (82,8\%,

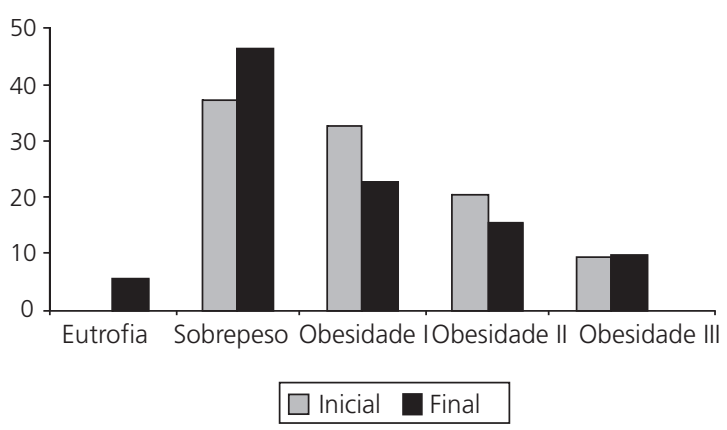

Figura 1. Distribuição, em porcentagem, dos participantes do PRAUSP, segundo estado nutricional inicial e final. Ribeirão Preto (SP), 2009.

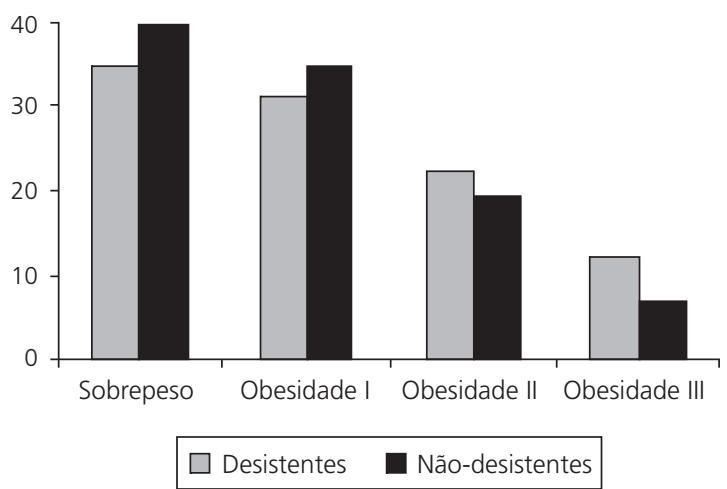

Figura 2. Distribuição, em porcentagem, dos participantes do PRAUSP, segundo grupo e estado nutricional. Ribeirão Preto (SP), 2009

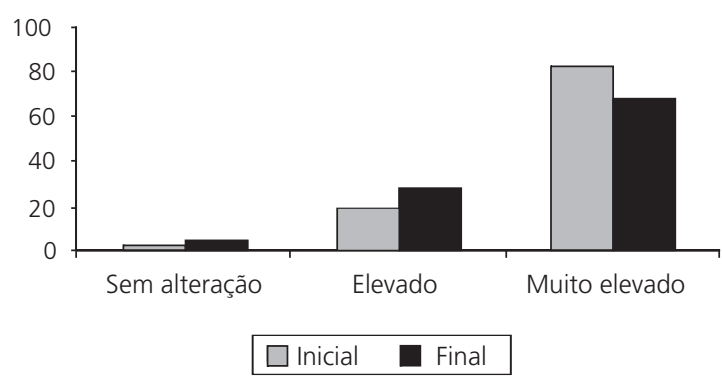

Figura 3. Distribuição, em porcentagem, dos participantes do PRAUSP, segundo o risco de complicações metabólicas relacionado à circunferência da cintura, no início e ao término do programa. Ribeirão Preto (SP), 2009.

$n=48)$, e que, apesar da redução significativa, permaneceu elevado ao final do programa $(67,2 \%$, $n=39$ ).

No decorrer da intervenção, a redução do IMC dos participantes provocou redução concomitante da circunferência da cintura, com relevância clínica e estatística $(p=0,00 ; r=0,46)$.

\section{DISCUSS ÃO}

O programa aqui descrito, denominado PRAUSP, consiste no oferecimento de orientação alimentar, apoio psicológico e prática regular de exercício físico para indivíduos com excesso de peso, visando a mudanças comportamentais que levem o emagrecimento adequado e sadio. Considerando que a obesidade é uma condição de difícil manejo, há necessidade de abordagem multidisciplinar e interprofissional que busque como estratégia principal a mudança no estilo de vida, com novos hábitos alimentares e maior gasto energético. Assim, a organização de ações integradas do programa citado se justifica por evidências recentes de que a combinação entre restrição alimentar e prática regular de exercícios físicos, associada à terapia de mudança comportamental, apresenta-se como prática efetiva no tratamento da obesidade ${ }^{14}$.

No presente estudo, houve adesão de $50 \%$ dos participantes; desses, a maior porcentagem era de indivíduos com obesidade graus II e 
III. Isso pode ser explicado pela dificuldade que pessoas mais obesas têm de enfrentar aspectos referentes à dieta e por fatores de ordem emocional.

Outros estudos mostram taxas elevadas de desistência, como o de DiMatteo ${ }^{15}$ : 75\% dos pacientes não seguiram as recomendações relacionadas às mudanças no estilo de vida, como restrições alimentares, abandono do fumo e outros. A adesão ao tratamento, medicamentoso ou não, é fundamental para o sucesso da terapia instituída pela equipe de saúde e envolve outros comportamentos inerentes à saúde, que vão além do simples seguimento da prescrição. Sendo assim, vários fatores podem influenciar a adesão ao tratamento e estão relacionados ao paciente (sexo, idade, etnia, estado civil, escolaridade e nível socioeconômico); à doença (cronicidade, ausência de sintomas e consequências tardias); às crenças de saúde, hábitos de vida e culturais (percepção da seriedade do problema, desconhecimento, experiência com a doença no contexto familiar e autoestima); ao tratamento dentro do qual se encontra a qualidade de vida (custo, efeitos indesejáveis, esquemas terapêuticos complexos), à instituição (política de saúde, acesso ao serviço de saúde, tempo de espera versus tempo de atendimento); e, finalmente, ao relacionamento com a equipe de saúde ${ }^{10}$.

A opinião comum de que os pacientes são unicamente responsáveis por seguir seu tratamento é enganadora e reflete o equívoco mais comum de como outros fatores afetam o comportamento e a capacidade da pessoa aderir a seu tratamento ${ }^{11}$.

Neste estudo, apesar de os indivíduos com excesso de peso procurarem o PRAUSP como estratégia terapêutica para perda de peso, provavelmente metade deles não estivesse pronta para uma ação efetiva para a mudança de hábitos e redução do peso. Para Chapman et al. ${ }^{16}$, a relação entre o que as pessoas sabem e o que elas fazem tem sido considerada como "altamente tênue", pois o conhecimento não instiga a mudança, mas pode funcionar como um instrumento quando os sujeitos desejam mudar.

A alta taxa de abandono encontrada neste estudo pode ser decorrente do fato de os inscritos no programa talvez se encontrarem nos estágios iniciais de mudança segundo o modelo transteorético ${ }^{17} \mathrm{e}$, portanto, não estarem preparados ou motivados para a ação, ou seja, para a mudança de hábitos e consequentemente para a redução de peso.

De qualquer forma, para aqueles que aderiram à estratégia, houve redução média de 3,9\% do peso, que mesmo não sendo expressa por redução na classe de IMC, foi significativa no IMC médio dos participantes. Também foi possível perceber redução do percentual de indivíduos obesos de $60,3 \%$ para $44,8 \%$ após a intervenção. De acordo com Beraldo et al. ${ }^{18}$, a orientação realizada pelo nutricionista, além de oferecer um planejamento dietético elaborado, oferece um plano de educação alimentar individualizado, visando à redução e/ou à adequação do peso corpóreo. Essa conduta, associada à prática de atividade física, como acontece no PRAUSP, são terapias de excelência para o tratamento do excesso de peso.

Uma limitação para a discussão desses resultados é a existência de poucas informações científicas sobre as repercussões de mudanças no estilo de vida em populações sem comorbidades. Essa situação é preocupante, pois segundo Fontaine et al. ${ }^{19}$, a obesidade tem impacto relevante na longevidade, e iniciativas com vistas à redução de peso trazem benefícios que podem ser observados em longo prazo, e que tendem a ser maiores quanto mais precoce for essa intervenção. Sendo assim, investigações sobre as repercussões da educação alimentar na saúde desses indivíduos seriam de grande relevância para orientar os profissionais de saúde envolvidos nessa linha de trabalho.

No presente estudo, além da perda ponderal média de 3,9\% após 10 semanas, 37,9\% dos indivíduos apresentaram perda maior ou igual a 5,0\%. Esses resultados são semelhantes aos 
encontrados por Barbato et al. ${ }^{20}$, que, avaliando a perda ponderal de adultos submetidos à orientação sobre dieta, exercício físico e comportamento alimentar, constataram que a perda ponderal média foi semelhante $(3,6 \%)$, porém em tempo mais curto (4 semanas). No entanto, apenas $21,0 \%$ dos sujeitos tiveram perda maior ou igual a 5,0\%. Para esses mesmos autores, esse índice (5,0\%) é efetivo para reduzir a pressão arterial, os níveis de colesterol total, LDL, renina plasmática, glicemia e insulinemia, trazendo benefícios para os perfis hemodinâmico, metabólico e neuroendócrino.

A educação alimentar é considerada eficaz no tratamento do excesso de peso, da síndrome metabólica e de outras doenças, sendo estratégia reconhecida por trabalhos nacionais e internacionais ${ }^{21}$. De acordo com Wood ${ }^{22}$, indivíduos que associam restrição calórica e exercício físico ao tratamento comportamental podem perder de $5,0 \%$ a $10,0 \%$ de seu peso basal em um período de quatro a seis meses. Sendo assim, pelos resultados obtidos em apenas 10 semanas do PRAUSP, pode-se pensar que uma maior porcentagem de indivíduos alcançaria a redução de $5,0 \%$ ou mais caso o programa se estendesse por mais tempo.

Mesmo que o porcentual de perda de peso esteja às vezes aquém do desejado pelo obeso, essa redução já é capaz de modificar favoravelmente diversas condições relacionadas à obesidade. Essa redução ponderal melhora a tolerância à glicose e o perfil lipídico, reduz os níveis de pressão arterial e os sintomas associados a doenças degenerativas articulares, depressão e apneia do sono, além de melhorar os escores que avaliam a qualidade de vida ${ }^{23}$.

No presente estudo, $82,8 \%$ dos participantes que aderiram ao programa tiveram risco muito elevado de complicações metabólicas no início; esse percentual, mesmo tendo sido reduzido significativamente durante o decorrer dos encontros, permaneceu em 67,2\%. Esses resultados são muito superiores aos encontrados por Olinto et al..24, que, ao avaliar 1935 adultos de
Pelotas, encontraram aproximadamente $40 \%$ das mulheres com risco muito elevado de complicações metabólicas e cerca de um terço dos homens com algum grau de elevação nesse risco.

Essa situação é muito preocupante: acredita-se que medidas elevadas da circunferência da cintura levam ao aumento no risco de complicações vasculares devido à heterogeneidade das propriedades metabólicas e localização anatômica dos adipócitos, o que levaria à resistência da ação da insulina25.

Os participantes do PRAUSP apresentaram melhora concomitante do IMC e da circunferência da cintura. Barbato et al. ${ }^{20}$, em seu estudo, também afirmam que a redução do peso se correlaciona fortemente com a diminuição da circunferência da cintura, sugerindo que a perda preponderante de gordura seria do tipo visceral. Assim, mesmo em situações em que não haja perda efetiva de peso, pode haver redistribuição da gordura total quando ocorre orientação nutricional adequada, refletindo, assim, na redução dessa medida.

\section{CONCLUSÃO}

Neste estudo, assim como em outros dessa natureza, encontrou-se pequena adesão dos indivíduos ao programa proposto. Essa alta desistência pode ser explicada por fatores intrínsecos ao indivíduo e ao tratamento, além de outros extrínsecos a essa relação.

A estratégia aplicada, não só para a redução de peso mas para os riscos relacionados à medida da circunferência da cintura e para a meIhora da qualidade de vida, mostrou-se eficiente no tempo de desenvolvimento do programa. Porém, poderia ser ainda mais benéfica se houvesse maior tempo de duração.

Dessa forma, novos estudos são necessários para ampliar o tema, já que são escassos os trabalhos que apresentam resultados de programas de educação alimentar em populações 
sem comorbidades e que abordam aspectos relacionados à adesão dos indivíduos ao tratamento proposto.

Por fim, é imprescindível uma busca ativa dos sujeitos desistentes para obter informações mais detalhadas dos motivos que os levaram ao abandono do programa, permitindo, assim, meIhor adequação do protocolo e da sua equipe executora às necessidades dessas pessoas.

\section{COLABORADORES}

J.M. BUENO participou da elaboração do trabalho, coleta e interpretação dos dados. F.S. LEAL participou da elaboração do artigo. L.P.L. SAQUY fez a coleta de dados. C.B. SANTOS fez a análise dos dados. R.P.P. RIBEIRO orientou o trabalho.

\section{REFERÊ N CIAS}

1. Pitanga FJG, Lessa I. Razão cintura-estatura como discriminador do risco coronariano de adultos. Rev Assoc Med Bras. 2006; 52(3):157-61.

2. World Health Organization. Global strategy on diet, physical activity and health: obesity and overweight. Geneva. [cited 2010 Aug. 25]. Available from: <http://apps.who.int/dietphysicalactivity/ publications/facts/obesity/en/>.

3. Instituto Brasileiro de Geografia e Estatística. Pesquisa de orçamento familiar (POF), 2002/2003. Rio de Janeiro. [acesso 2010 ago 25]. Disponível em: $<$ http://www.ibge.gov.br>.

4. Roth J, Qiang X, Marbán SL, Redelt H, Lowell $B C$. The obesity pandemic: where have we been and are we going? Obes Res. 2004; 12(2):88-100.

5. Wing RR, Hill JO. Successful weight loss maintenance. Annu Rev Nutr. 2001; 21(1):323-41. doi: 10.1146/annurev.nutr.21.1.323.

6. Garcia RWD. Um enfoque simbólico do comer e da comida nas doenças. Rev Nutr. 1992; 5(1):70-80.

7. Rotenberg S, Vargas S. Práticas alimentares e o cuidado da saúde: da alimentação da criança à alimentação da família. Rev Bras Saúde Mater Infant. 2004; 4(1):85-94. doi: 10.1590/51519-38 292004000100008.

8. Sturmer JS. Reeducação alimentar: qualidade de vida, emagrecimento e manutenção da saúde. $5^{a}$ ed. Petrópolis: Vozes; 2001.
9. Cuvello LC, Patin R. Restrição versus reeducação alimentar. In: Damaso A, coordenadora. Obesidade. Rio de Janeiro: Medsi; 2003. p.367-74.

10. Boog MCF. Educação nutricional: passado, presente e futuro. Rev Nutr. 1997; 10(1):5-19.

11. Gusmão JL, Mion Jr D. Adesão ao tratamento: conceitos. Rev Bras Hipert. 2006; 13(1):23-25.

12. Haynes RB. Determinants of compliance: the disease and the mechanics of treatment. In: Haynes RB, Taylor DW, Sackett DL.Compliance in healthcare. Baltimore (MD): Johns Hopkins University Press; 1979. p.49-62.

13. Rand CS. Measuring adherence with therapy for chronic diseases: implications for the treatment of heterozygous familial hypercholesterolemia. Am J Cardiol. 1993; 72:68-74.

14. Brasil. Ministério da Saúde. Vigilância Alimentar e Nutricional. SISVAN: orientações básicas para a coleta, processamento, análise de dados e informação em serviços de saúde. Brasília: Ministério da Saúde; 2004. p.118.

15. Statistical Package for the Social Sciences Incorporation. SPSS for Windows. Release 16.1. Chicago: SPSS Inc.: 2007.

16. Ross R, Janssen I, Dawson J, Kungl AM, Kuk JL, Wong $\mathrm{SL}$, et al. Exercise-induced reduction in obesity and insulin resistance in women: a randomized controlled trial. Obes Res. 2004;12(5):789-98. doi: 10.1038/oby.2004.95.

17. Dimatteo MR. Enhancing patient adherence to medical recommendations. J Am Med Assoc.1994; 271(1):79-83.

18. Chapman KM. Appeying behavioral models to dietary education of eldery diabetic patients. J Nutrl Educ. 1995; 27(2):75-9.

19. Prochaska JO, Redding CA, Evers KE. The transtheoretical model and stages of change. In: Glanz K, Rimer BK, Viswamath K, editors. Health behavior and health education: theory, research and practice. San Francisco Jossey: Bass Publishers; 1997 p.60-84.

20. Beraldo FC, Vaz IMF, Naves MMV. Nutrição, atividade física e obesidade em adultos: aspectos atuais e recomendações para prevenção e tratamento. Rev Med Minas Gerais. 2004; 14(1):57-62.

21. Fontaine KR, Redden DT, Wang C, Westfall AO, Allison DB. Years of life lost due to obesity. JAMA; 2003; 289(2):187-93.

22. Barbato KBG, Martins RCV, Rodrigues MLG, Braga JU, Francischetti EA, Genelhu VA. Efeitos da redução de peso superior a $5 \%$ nos perfis hemodinâ- 
584 | J.M. BUENO et al.

mico, metabólico e neuroendócrino de obesos grau I. Arq Bras Cardiol. 2006; 87(1):12-21. doi: 10.15 90/S0066-782X2006001400003.

23. Klack K, Carvalho JF. A importância da intervenção nutricional na redução do peso corpóreo em pacientes com síndrome do anticorpo antifosfolípide. Rev Bras Reumatol. 2008; 48(3):134-40.

24. Wood AJJ. Obesity. N Engl J Med. 2002; 346 (8): 591-601.

25. Blumenthal JA, Sherwood A, Gullette EC. Exercise and weight loss reduce blood pressure in men and women with mild hypertension: effects on cardiovascular, metabolic, and hemodynamic functioning. Arch Intern Med. 2000; 160(13): 1947-58.

26. Olinto MTA, Nacul LC, Dias-da-Costa JS, Gigante DP, Menezes AM, Macedo S. Níveis de intervenção para obesidade abdominal: prevalência e fatores associados. Cad Saúde Pública. 2006; 22(6): 1207-1215.

27. Bray GA. Medical consequences of obesity. J Clin Endocrinol Metab. 2004; 89(6):2583-9.

Recebido em: 29/10/2009

Versão final reapresentada em: 29/11/2010 Aprovado em: 10/1/2011 